\title{
Response: "Another merit of fetal MRI in prenatal diagnosis of right aortic arch"
}

\author{
Gülen Yerlikaya ${ }^{1}$ (1)
}

Received: 2 April 2019 / Accepted: 11 April 2019 / Published online: 1 May 2019

(c) Springer-Verlag GmbH Germany, part of Springer Nature 2019

Matsubara et al. described the possibility to detect the presence or absence of tracheal stenosis in fetal MRI [1]. Prenatal diagnosis of airway obstruction is important in terms of postnatal management and outcome. Unfortunately, we were not able to confirm the presence of this signs in the two cases, who presented airway obstruction postnatally, in our recent study. However, fetal MRI is becoming increasingly important in diagnosing fetal abnormalities and the ability to observe tracheal stenosis shows the high sensitivity to detect pathological features.

As mentioned before, foetal MRI becomes more common as part of prenatal diagnosis, if available. Indeed, it is very useful to see additional fetal features, which may not be clear in the ultrasound examination, especially when fetal echocardiography is limited [2]. It shows high sensitivity (95.6\%) in the diagnosis of aortic arch abnormalities and it is claimed to be the best modality for demonstrating the arch vessels [3, 4]. Regarding the diagnosis of possible airway obstruction in cases of right aortic arch, as described by Matsubara et al. [1], it is a very interesting new finding, which should be paid more attention too, when performing foetal MRI with suspicion of vascular ring malformations. As a consequence, it may be used as a prognostic sign, whether tracheal obstruction will be symptomatic after birth and need further treatment or even intervention in new-borns. Thus, the presence or absence of this specific morphological features may help to improve parental counselling and postnatal management.

Nevertheless, although MRI is a promising tool in cardiac abnormalities and aortic arch malformations, the importance of ultrasound and foetal echocardiography should not be denied. Furthermore, recommendation for karyotyping should be offered in any case of (right) aortic arch abnormalities, irrespective of co-existing malformations and availability of foetal MRI.

\section{Compliance with ethical standards}

Conflict of interest The author declares that there is no conflict of interest and received no funding regarding this study.

\section{References}

1. Matsubara D, Takahashi H, Kataoka K, Minami T, Furukawa R, Matsubara S, Yamagata T (2018) Tracheal stenosis due to vascular rings: its possible prenatal diagnosis based on four cases of vascular rings with or without eventual tracheal stenosis. Clin Exp Obstet Gynecol (Epub ahead of print)

2. Dong SZ, Zhu M, Li F (2013) Preliminary experience with cardiovascular magnetic resonance in evaluation of fetal cardiovascular anomalies. J Cardiovasc Magn Reson 21(15):40

3. Zidere V, Tsapakis EG, Huggon IC, Allan LD (2006) Right aortic arch in the fetus. Ultrasound Obstet Gynecol 28:876-881

4. Li X, Li X, Hu K, Yin C, (2017) The value of cardiovascular magnetic resonance in the diagnosis of fetal aortic arch abnormalities. J Matern Fetal Neonatal Med 30(11):1366-1371

Publisher's Note Springer Nature remains neutral with regard to jurisdictional claims in published maps and institutional affiliations.

Gülen Yerlikaya

guelen.yerlikaya@meduniwien.ac.at

1 Division of Obstetrics and Feto-Maternal Medicine, Department of Obstetrics and Gynaecology, Medical University of Vienna, WaehringerGuertel 18-20, 1090 Vienna, Austria 